\title{
EL USO DE LA PALABRA EN LOS INFORMATIVOS RADIOFÓNICOS
}

\author{
Silvia Jiménez Martín \\ silviajimenezmartin@yahoo.es
}

\section{Resumen:}

En este trabajo de investigación analizamos el uso de la palabra en los informativos principales de mediodía de los noticiarios con más audiencia del país: Cadena Ser, Onda Cero, COPE y Radio Nacional. Se trata de un estudio formal que trata de medir el grado de expresividad -de atractivo- de los noticiarios que hemos relacionado con las diferentes modalidades de palabra que encontramos en los informativos: número de informaciones, duración de las mismas, tiempo de palabra utilizado por los editores, uso de la palabra por parte de los redactores y redactoras y número y duración de las grabaciones sonoras utilizadas.

Palabras claves: Palabra, elementos, testimonios, redactores, creatividad, expresividad, alternancia, riqueza.

\begin{abstract}
:
In this work of research we analyze the use of the word in the informative principal ones of midday of the newscasts with more hearing of the country: Cadena Ser, Onda Cero, Cadena Cope y Radio Nacional. It is a question of a formal study that it(he,she) tries to measure the degree of expressiveness - of attraction - of the newscasts that we have related to the different modalities of word that we find in the informative ones: number of information, duration of the same ones, time of word used by the publishers(editors), use of the word on the part of the editors and editors and number and duration of the sonorous used recordings.
\end{abstract}

Keywords: Word, elements, testimonies, editors, creativity, expressiveness, alternation, richness.

\section{INTRODUCCIÓN}

E I objetivo de este trabajo es analizar el uso de la palabra hablada en los informativos radiofónicos nacionales. Partimos de la hipótesis de que los editores priorizan este elemento del lenguaje radiofónico frente al resto, restando expresividad a los noticiarios y disminuyendo la atención del oyente por crear productos poco atractivos que resultan monótonos y repetitivos para la audiencia. Partimos de la idea de que en la comunicación radiofónica -sea del tipo que sea- los mensajes valiosos son aquellos que consigue comunicar con eficacia. La eficacia comunicativa 
la relacionamos con dos conceptos: con las habilidades del radiofonista, que se reflejan inexorablemente en el producto informativo, y con el modo de recibir el mensaje, es decir, con la atención que presta el oyente.

En primer lugar, consideramos que para que un mensaje resulte efectivo tiene que ser expresivo, según el DRAE, manifestar con viveza lo que se piensa. Sin embargo, resulta poco posible cuantificar la expresividad que encierra cada término, aunque sí podemos medir el tiempo que dedican los periodistas a cada una de las manifestaciones en las que se presenta el elemento objeto de estudio -la palabra informativa-, vinculando de este modo la comunicación eficaz con la expresividad formal. Por lo tanto, la investigación que presentamos es un estudio de formas -uso de la palabra en la estructura del informativo- y no de contenidos, difícilmente mesurables. Desde el punto de vista formal, y en relación con el uso de los elementos del lenguaje radiofónico, la expresividad de los informativos es directamente proporcional a la combinación de los cuatro elementos del lenguaje: palabras, músicas, efectos sonoros y silencios.

Si atendemos sólo al uso de la palabra, para reforzar la viveza y eficacia comunicativa, es decir, para mejorar la expresividad, debemos atender a factores que supongan una renovación del estímulo auditivo. Para ello, hemos de fijarnos en el número de informaciones (cuantas más se introduzcan mejor), y en la brevedad de las mismas, en un uso correcto, no excesivo, de la palabra por parte del editor, en un alto y equilibrado número de redactores y redactoras. Por último, incrementar el número de documentos sonoros de no demasiada duración también nos ayudará a mejorar la expresividad:

\begin{abstract}
La importancia de combinar la palabra de los periodistas con las declaraciones de los implicados en la información tiene una doble raíz. Por un lado, las características de la radio como medio basado exclusivamente en el sonido imponen la combinación de voces para mantener la atención del oyente. Con ello se consigue una información más dinámica, con más ritmo, una información que elimina la monotonía del discurso único. Por otro lado, la variedad de voces es importante ya que permite conocer al oyente otro punto de vista que no sea el del periodista (Rodero y Merayo, 1997: 27).
\end{abstract}

En segundo lugar, hemos vinculado la eficacia informativa con la atención que presta el oyente, tratando de estudiar dónde descansa la estética -el atractivo- del medio. En este sentido, y, aludiendo a las características psico-físicas del individuo, Pérez (2005) indica que cuanto más atractivo sea el mensaje desde el punto de vista formal, más atención se espera del oyente. Así pues, la atención también depende de las características formales y, como indica Soengas (2003) el atractivo está estrechamente vinculado al grado de expresividad, con independencia del tipo de programa:

Es un error pensar que sólo la función semántica es propia de los programas informativos y que la función estética es exclusiva de los espacios dramáticos o los de creación. Todos los programas han de ser interesantes y atractivos porque en la información también tiene cabida la expresividad (p. 215). 
A nuestro juicio, las formas atractivas deben incluirse en los informativos puesto que no están reñidas con los contenidos interesantes ni son más propias de unos programas que de otros. Los noticiarios precisan de una renovación formal que priorice el fin para el que se construyen. No informa quien no capta la atención de la audiencia. Y no informa quien presenta mensajes que no se escuchen. Así las cosas, consideramos que el grado de expresividad de los informativos está estrechamente relacionado con su atractivo y que ambos repercuten en la atención que prestan los oyentes al mensaje, de manera que podemos utilizar estos dos términos indistintamente.

Para comprobar el abuso de la palabra como generadora de imágenes frente a otros recursos expresivos, en la parte central de la investigación hemos realizado un exhaustivo análisis de la misma, midiendo segundo a segundo su aparición en los principales informativos de noticias de mediodía de las cuatro emisoras de más audiencia del país: Hora 14 -Cadena Ser-, La Palestra -COPE-, Las Noticias de Mediodía -Onda Cero- y Diario Directo -Radio Nacional. El estudio se ha realizado mediante grabaciones, resultado de un muestreo aleatorio no probabilístico, cuya unidad la conformaron los días laborables del mes de noviembre de 2005. De los informativos de las cuatro cadenas se seleccionaron 30 minutos consecutivos de información nacional entre las dos y las tres de la tarde, aunque no coincidieron los horarios elegidos en las cuatro cadenas porque cada una destina uno diferente a la información local y regional.

Para la medición de las variables palabra se elaboró una plantilla en la que recogimos diversos parámetros para su análisis en las tres partes de los informativos: apertura, desarrollo y cierre. En primer lugar, anotamos los resultados individuales obtenidos en cada cadena y después los comparamos. Las variables elegidas para la medición fueron las siguientes:

\section{Número y duración de las informaciones.}

2. Tiempo del editor: compuesto por el tiempo dedicado al saludo y presentación del programa, la presentación de las noticias de los redactores y el cierre ${ }^{1}$ o despedida.

3. Tiempo de los redactores: distinguimos entre redactores masculinos y femeninos.

4. Tiempo de las grabaciones sonoras: anotamos tanto el número como la duración de las mismas.

A continuación reflejamos los resultados obtenidos en cada una de las partes, vinculándolos con el grado de expresividad y de eficacia comunicativa.

\footnotetext{
${ }^{1}$ El cierre de los informativos no es un cierre literal, puesto que en tres de las cuatro emisoras grabadas la finalización del tiempo de grabación no coincidió con el final de programa, de manera que no nos referiremos al cierre de manera estricta, que sólo se produce como tal en RNE -esta cadena tiene su despedida a las tres del mediodía, la misma hora a la que finalizó la grabación-, sino a un cierre figurado, con el que queremos reflejar los últimos minutos del programa.
} 


\section{NÚMERO Y DURACIÓN DE LAS INFORMACIONES}

El tiempo denominado información engloba el total de los segundos dedicados a la palabra. Bajo este epígrafe se ha medido el tiempo ocupado por este elemento tras sumar el uso de la palabra por parte del editor, de los redactores y el ocupado por los cortes de sonido.

Consideramos que tanto el número de informaciones como su duración media influyen en el grado de expresividad de los programas, que ganan en agilidad y dinamismo cuantas más informaciones se incluyan y más breves sean. El siguiente cuadro recoge una comparación de los resultados obtenidos en las diferentes cadenas en este epígrafe.

Cuadro 1. Número de informaciones y tiempo medio de duración.

\begin{tabular}{|c|l|c|}
\hline \multirow{2}{*}{ EMISORAS } & \multicolumn{2}{|c|}{ INFORMACIONES } \\
\cline { 2 - 3 } & $\mathrm{N}^{\circ}$ & $\mathrm{T}(\mathrm{M})$ \\
\hline SER & 85 & 89,58 \\
\hline COPE & 79 & 97,17 \\
\hline ONDA CERO & 90 & 78,87 \\
\hline RNE & 90 & 95,87 \\
\hline
\end{tabular}

Fuente: Elaboración propia.

En total, el tiempo dedicado a la palabra en cada emisora ha sido el que sigue: en la Ser, 7.615 segundos; en Onda Cero, 7.099 segundos; en la Cadena COPE, 7.677 segundos y en Radio Nacional, 8.629 segundos. Los resultados de las cuatro cadenas aparecen recogidos en el siguiente gráfico:

Gráfico 1. Tiempo dedicado a la palabra.

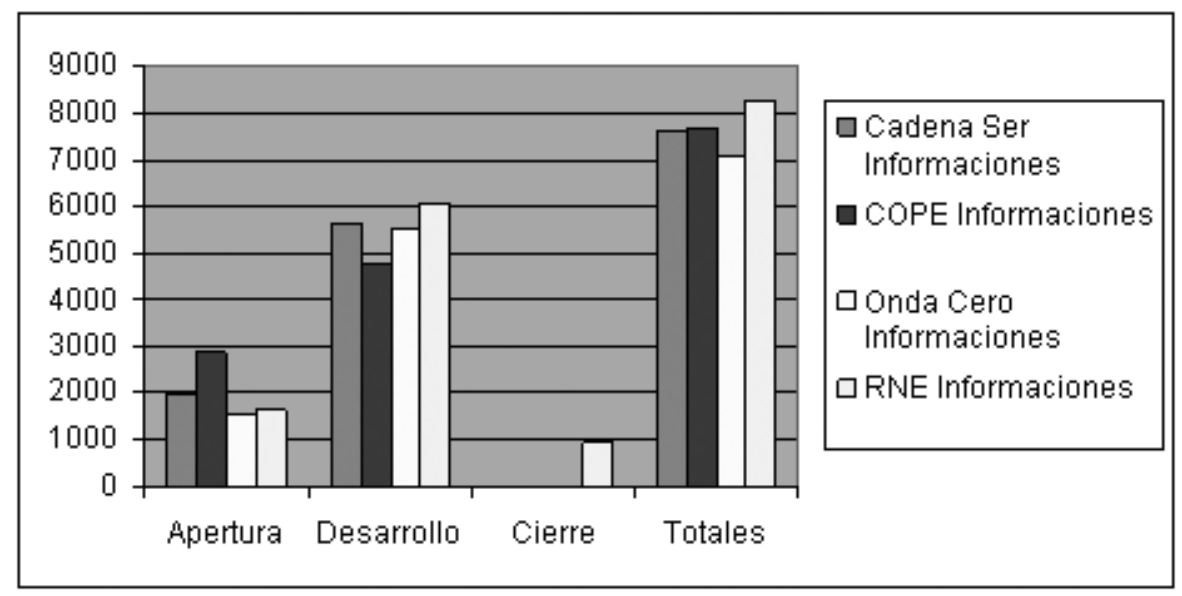

Fuente: Elaboración propia. 
El análisis de los resultados manifiesta que la cadena que más tiempo ha dedicado a la palabra ha sido RNE, seguida de la COPE, la Ser y Onda Cero. Sin embargo, los datos cambian si atendemos al tiempo medio de cada información, uno de los factores de los que depende la expresividad: las informaciones que han registrado una duración más extensa son las de la Cadena COPE, con un tiempo medio de 97,17 segundos y un total 79 informaciones; después se sitúan las de RNE con 95,87 y 90 piezas, seguidas de las la Cadena Ser con 89,58 segundos de media para 85 informaciones y, por último, Onda Cero, con una duración media por información de 78,87 y un total de 90 informaciones.

La cadena que mejor se adecua al criterio de brevedad -el tratamiento de las noticias ha de ser breve porque el medio exige esta característica- es Onda Cero, seguida de la Ser, Radio Nacional y la Cadena COPE. Onda Cero es, por lo tanto, la emisora más expresiva en este epígrafe.

\section{TIEMPO DE LOS EDITORES}

A continuación medimos el tiempo de los informativos que los oyentes emplearon en escuchar a los editores de cada una de las cadenas. En el gráfico se recogen los segundos que los editores están en posesión de la palabra, resultado de sumar los valores: presentación o saludos, pasos para introducir a los redactores/as y cierres o despedida.

Gráfico 2. Tiempo de los editores.

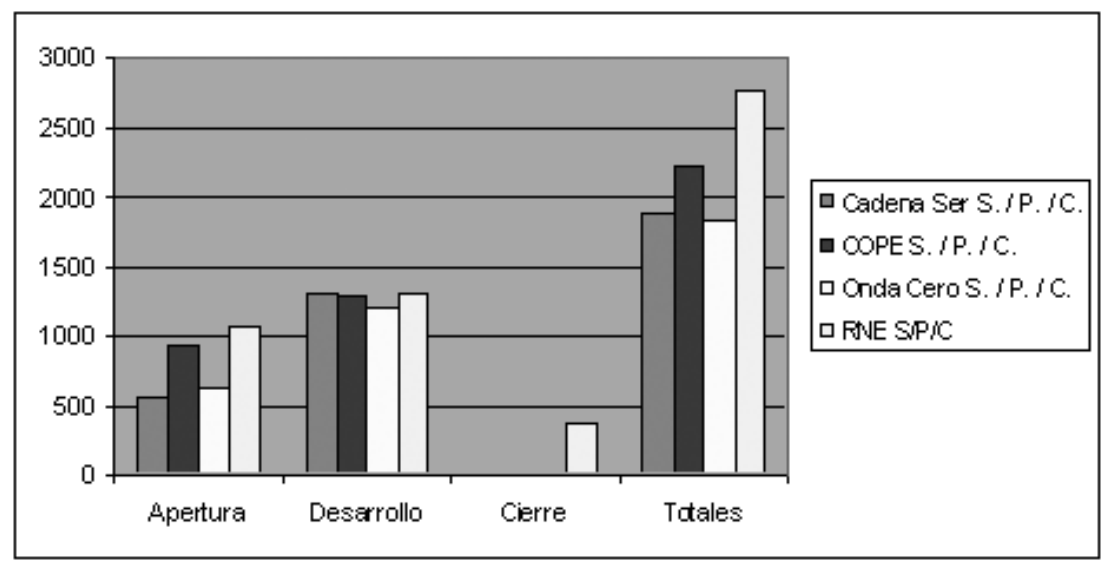

Fuente: Elaboración propia.

RNE ha sido la emisora en la que los editores han estado más tiempo en posesión de la palabra, un dato que nos indica que son los informativos en los que se observa de un modo más evidente la personalidad de los responsables. De todos modos, como los informativos de la radio pública no programan publicidad, su tiempo ha sido superior al de otras emisoras. El segundo informativo con más presencia del editor ha sido el de la COPE. Sin embargo, existe otro dato que nos confirma que RNE ha sido la cadena más expresiva en este apartado: ha sido la única que se des- 
marca del resto al elegir a dos editores principales - uno de cada sexo, por lo que la alternancia de voces a lo largo del programa queda garantizada. Este hecho supone que los informativos de esta emisora sean más ricos en pluralidad y en originalidad.

En el reparto del tiempo observamos que es en la apertura de todas las cadenas donde los editores están más tiempo en posesión de la palabra. En general, sus voces son agradables -sobre ellos recae todo el peso del programa-y prestan atención a la locución en la presentación para facilitar la comprensión del mensaje. En todos los casos, anotamos rasgos de un buen locutor como son naturalidad, claridad, una correcta pronunciación, buen ritmo y velocidad de lectura y una entonación adecuada.

\section{TIEMPO DE LOS REDACTORES}

En nuestra opinión, la expresividad depende también del número de voces que aparecen en el programa y de la correcta combinación de timbres -voces femeninas intercaladas con voces masculinas. El siguiente cuadro muestra el número de redactores que intervinieron en los programas (V), el número de redactoras (M) y la suma de ambos $(\mathrm{T})$

Cuadro 2. Número de redactores.

\begin{tabular}{||c||c||c||c||}
\hline \multirow{2}{*}{ EMISORAS } & \multicolumn{3}{c||}{ REDACTORES } \\
\cline { 2 - 4 } & $\mathrm{V}$ & $\mathrm{M}$ & $\mathrm{T}$ \\
\hline \hline Cadena Ser & 41 & 34 & 75 \\
\hline \hline COPE & 33 & 34 & 67 \\
\hline \hline Onda Cero & 37 & 32 & 69 \\
\hline \hline RNE & 50 & 29 & 79 \\
\hline \hline
\end{tabular}

Fuente: Elaboración propia.

Para que resulte más visible la comparación de los resultados aportamos dos gráficos comparativos.

Gráfico 3. Voces masculinas.

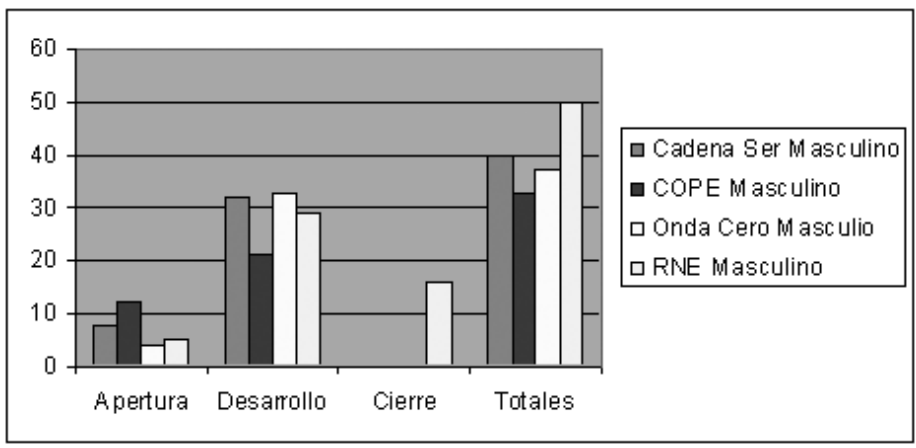

Fuente: Elaboración propia. 
Gráfico 4. Voces femeninas.

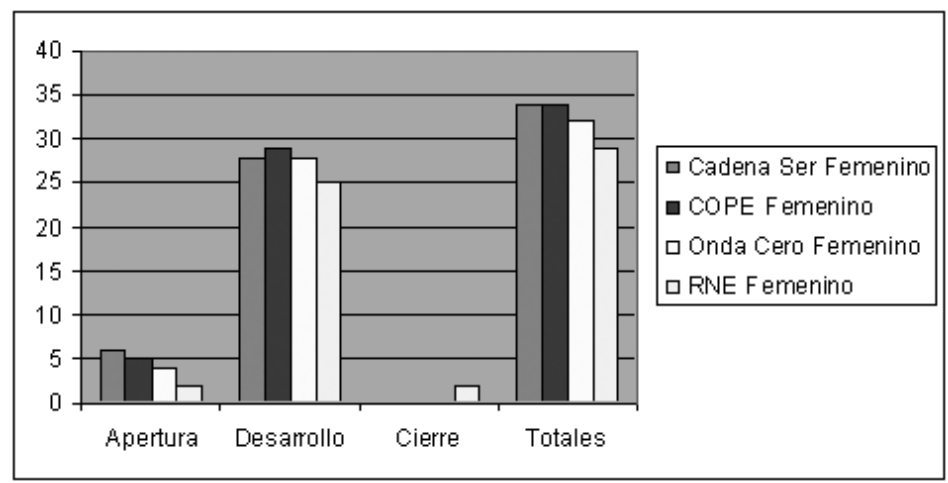

Fuente: Elaboración propia.

Los profesionales de las distintas emisoras son quienes se reparten el peso del programa. A nuestro juicio, este dato resulta positivo porque supone un cambio de voces. Con la introducción de más redactores se garantiza una mayor credibilidad, al consultar a la fuente más cercana al suceso. La COPE es la única emisora en la que prácticamente se alcanza el equilibrio entre el número de redactores masculinos y femeninos (33-34). La óptima combinación de voces ha dado más color a los programas de esta emisora. Por lo tanto, podemos asegurar que es la más expresiva en este apartado. Por otro lado, debemos destacar que es la única en la que se concede prioridad a las voces femeninas.

La segunda emisora más expresiva en lo que respecta al empleo de voces es Onda Cero, donde el número de varones y mujeres, aunque aparece descompensado, no alcanza los niveles de desequilibrio que en la Cadena Ser, la tercera menos expresiva de las analizadas.

El último puesto lo ocupa RNE, la cadena en la que más desigualados están los datos de los redactores masculinos con respecto a los femeninos, siendo el número de varones casi el doble que el de mujeres (50-29). Sin embargo, a favor de la emisora hay que indicar que esta descompensación se debe a la desigualdad que se produce en el último tramo del programa, dedicado a los deportes. Hasta que este tipo de contenidos entra en escena, el número de voces masculinas y femeninas estaba prácticamente igualado. A favor de RNE también hay que señalar que es la emisora que más redactores ha incluido de las cuatro. Si tenemos en cuenta que el número de voces que intervienen en un programa está relacionado con la amenidad del mismo, podemos asegurar que, pese a las diferencias que apuntábamos, los programas de RNE han resultados amenos.

Para el análisis del índice de variabilidad ${ }^{2}$, consideramos que la emisora más expresiva es la que se aproxime al uno -un redactor por cada noticia-. Así, el primer puesto es para la Ser $(0,88)$, seguida de RNE $(0,87)$.

\footnotetext{
${ }^{2}$ En este caso el índice de variabilidad es el resultado de dividir el número total de redactores entre el número de informaciones, para saber si todas las noticias fueron leídas por algún redactor.
} 
En general, las voces de todos los redactores/as resultan agradables, a pesar de que en las grabaciones hemos percibido que la entonación de algunos/as no siempre fue la correcta. En ocasiones, percibimos un defecto frecuente en este tipo de programas: el efecto cántico.

\section{TIEMPO DE LOS TESTIGOS}

El número de testimonios insertados por una cadena, además de conceder credibilidad al discurso, supone un apoyo formal -provocado por la renovación del estímulo sonoro- que ayuda a captar la atención del oyente. Así, el grado de expresividad del programa depende directamente del número de testimonios que se inserten y de que la duración de éstos no sea demasiado elevada, que preferiblemente no supere el tiempo medio estandarizado de 20 segundos.

Los resultados obtenidos en el estudio reflejan que el tiempo que la cadena destina a los cortes de sonido es el siguiente: la Ser, 1.660 segundos; Onda Cero, 2.212 segundos; la COPE, 1.606 segundos y RNE: 1.807. Otro dato analizado es el número total de testimonios que escuchamos en cada cadena y su duración media expresada en segundos, que quedan registrados en la siguiente tabla.

Cuadro 3. Tiempo de los testigos.

\begin{tabular}{||c||c||c||}
\hline \multirow{2}{*}{ EMISORAS } & \multicolumn{2}{c||}{ TESTIMONIOS } \\
\cline { 2 - 3 } & $N^{\circ}$ & $\mathrm{T}(\mathrm{M})$ \\
\hline \hline Cadena Ser & 97 & 17,11 \\
\hline \hline COPE & 68 & 23,61 \\
\hline \hline Onda Cero & 105 & 20,11 \\
\hline \hline RNE & 77 & 23,46 \\
\hline \hline
\end{tabular}

Fuente: Elaboración propia.

Por otro lado, para poder comparar los resultados obtenidos en cada emisora con los del resto, nos hemos apoyado en un índice, que hemos denominado de variabilidad, resultante de dividir el número de cortes de sonido entre el total de informaciones, con el fin de determinar cuántas grabaciones acompañan a cada información. El índice de variabilidad obtenido de la Ser es de 1,14. Como el índice es superior a la unidad, deducimos que todas las informaciones han estado documentadas; lo mismo sucede en Onda Cero, con un índice de variabilidad del 1,16. Sin embargo, ni la COPE, con 0,86, ni en RNE, con 0,85, este índice supera la unidad.

A continuación, observamos en el gráfico con los resultados comparativos por cadenas. 
Gráfico 5. Tiempo de los testigos.

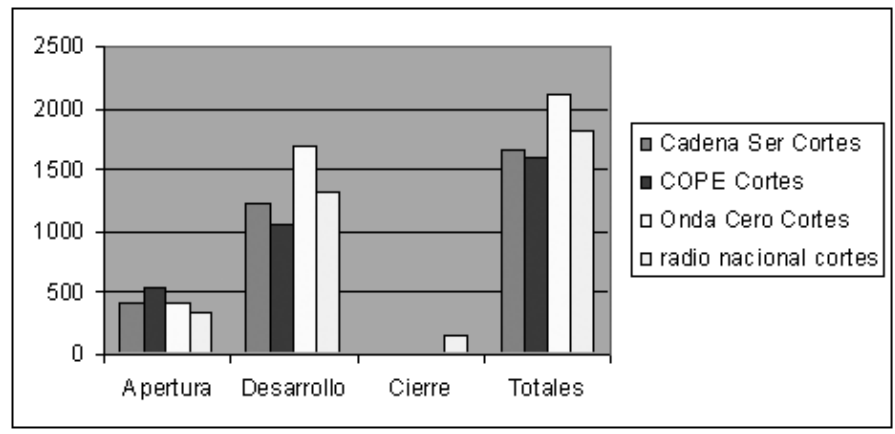

Fuente: Elaboración propia.

Onda Cero fue la emisora que introdujo un mayor número de grabaciones sonoras en términos absolutos, con un total de 105, seguida de la Ser, con 97 . Sin embargo, si atendemos al índice de variabilidad, la que más cortes introdujo fue la Cadena Ser, seguida de Onda Cero; ambas son las dos únicas emisoras en las que este índice supera la unidad. Con respecto a la duración de los mismos, en la Ser son más breves, más dinámicos, con una media de 17,11 segundos, mientras que en Onda Cero la duración media de los documentos asciende a 20,11.

La Cadena Ser consigue ventaja en este epígrafe con respecto a las demás, puesto que inserta más documentos en sus informaciones y son más breves, con lo que se recupera continuamente la atención del oyente. La emisora que menos cortes introdujo tanto en términos absolutos como en términos relativos fue la COPE, la menos expresiva de las cuatro en este apartado. Además, sus cortes de sonidos son más extensos, alcanzando una media de 27,42 segundos.

\section{CONCLUSIONES}

Los resultados del análisis de los informativos principales de mediodía de las cuatro cadenas de radio más importantes del país revelan, que, en líneas generales, los noticiarios priman el valor semántico sobre el expresivo.

Las palabras, como base del discurso, deben estar acompañadas y reforzadas por el resto de elementos para alcanzar su máximo nivel comunicativo. Sin embargo, en los informativos estudiados se les ha restado toda la potencialidad que podrían alcanzar en el medio radiofónico, reduciendo extraordinariamente sus posibilidades. Sin la combinación de elementos, no se refuerza la expresividad.

Onda Cero ha combinado mejor que ninguna los criterios de cantidad y duración de las informaciones. Se da la circunstancia de que ha sido la cadena que ha introducido el mayor número de informaciones en menos tiempo. La Ser ha registrado el segundo mejor puesto, aunque que el tiempo medio de sus informaciones ha sido excesivo. Por último, tanto las informaciones en la COPE como las de RNE han sido excesivamente largas, ya que en ambos casos el tiempo de duración media se aproxima a los cien segundos. 
La mayor parte del tiempo destinado a la palabra en la apertura de los programas lo ocupan los editores, mientras que en el desarrollo de los mismos cobran más importancia los redactores. Por lo que respecta a los editores, la cadena más plural y más expresiva ha sido RNE. Es la única que en la que aparecen dos editores principales - uno de cada sexo-, que garantizan la alternancia de voces a lo largo del programa. Además, en el segmento final aparece un nuevo editor masculino que refuerza aún más el cambio de ritmo en el programa. Por todo, RNE ha sido la emisora en la que los editores han tenido más protagonismo y la que posee unos informativos más personalizados, con más sello e impronta que los del resto de emisoras. Los segundos informativos en los que el editor tiene más gancho son los de la COPE, que introduce dos editores, aunque la femenina sólo se encarga del apartado de noticias internacionales en el desarrollo del programa. La Cadena COPE es la única emisora en que prácticamente se alcanza el equilibrio entre el número de redactores masculinos y femeninos. La óptima combinación de voces ha dado más color a los programas de esta emisora. Por lo tanto, es la cadena más expresiva en este apartado. Por otro lado, destacar que es la única en la que se concede prioridad a las voces femeninas. El resto de cadenas sólo introducen un editor.

Los datos son favorables en lo que se refiere a la alternancia de voces, aunque, según nuestro concepto de expresividad, faltan redactores en todos los programas. La combinación de timbres ha aportado a los informativos dinamismo y amenidad.

En general, a través de los índices de variabilidad, que determinan el número de redactores por cada información, detectamos una carencia de profesionales en todos los informativos, puesto que en ningún caso los valores obtenidos alcanzan la unidad, siendo la Cadena Ser la que cuenta con más redactores en términos relativos y RNE la que posee más redactores en términos absolutos. Sin embargo, en el último tramo del programa de la emisora pública la mayoría de los redactores son varones, de manera que, la diferencia entre éstos y el número de redactores femeninos aumenta considerablemente en el cómputo total.

Los datos no son positivos para todas las emisoras en lo que se refiere al número de grabaciones sonoras utilizado, bastante escaso, ya que no siempre las informaciones que ofrecen los noticiarios principales del día están documentadas. A nuestro juicio, este hecho resta ritmo al informativo, dificulta la atención del oyente y disminuye la credibilidad y la fiabilidad. Para obtener los datos relacionados con los documentos sonoros nos hemos basado en un índice de variabilidad que nos ha permitido comparar el número de grabaciones por información. Los peores resultados son para RNE y la COPE, cuyo índice de variabilidad no alcanza la unidad, lo que significa que no todas las informaciones de estas cadenas estuvieron ilustradas con testimonios. La emisora que menos cortes introdujo tanto en términos absolutos, como en términos relativos fue la COPE, la menos expresiva de las cuatro en este apartado.

Con respecto a la utilización de documentos sonoros, los informativos de la Cadena Ser son los más expresivos, ya que introducen un número importante de voces de más testigos. En términos relativos, el índice de variabilidad supera la unidad y todas las informaciones están documentadas con cortes de voz breves y dinámicos. El segundo lugar lo ocupa Onda Cero, que es la única emisora en la que escucha- 
mos más tiempo a los protagonistas de la información que al propio presentador del programa.

A tenor de todo lo expuesto, consideramos que la emisora más expresiva en el uso de la palabra ha sido la Cadena Ser, por ser la que más indicadores favorables reúne: es la emisora con mayor presencia de redactores, si relacionamos el dato con el número de informaciones por profesional y de documentos sonoros, en términos relativos. Por otro lado, sus grabaciones son más breves que en el resto de cadenas y sus informaciones son las segundas menos extensas.

\section{REFERENCIAS BIBLIOGRÁFICAS}

JIMÉNEZ, S. El proceso de elaboración de informativos radiofónicos. Tesis Doctoral inédita. Universidad Pontificia de Salamanca, 2006.

PÉREZ, A. El proceso de comprensión auditiva. Estrategias comunicativas aplicadas a los boletines horarios. Tesis Doctoral inédita, Universidad Pontificia de Salamanca, 2005.

RODERO, E.; MERAYO, A. La información radiofónica en Castilla y León. Análisis de los informativos regionales. Valladolid: Junta de Castilla y León, 1997.

SOENGAS, X. Informativos radiofónicos. Madrid: Cátedra, 2003.

\section{Breve semblanza de la autora}

Silvia Jiménez Martín es Doctora en Comunicación por la Universidad Pontificia de Salamanca. Profesora invitada en las Facultades de Comunicación Social y de Locución Nacional de la UCES, y en la Carrera de Producción y Creatividad Radiofónica en la Escuela Terciaria ETER, ambas en Buenos Aires. Ha impartido clases de radio en las titulaciones de Publicidad y Relaciones Públicas y Periodismo en la Universidad San Jorge (Zaragoza) y como colaboradora honorífica en la Universidad Complutense. Como profesional, destaca su labor informativa en Onda Madrid. Asimismo, ha dirigido y presentado magazines locales en las cadena Ser, COPE y Onda Cero y ha trabajado en periódicos diarios y en prensa y revistas especializadas.

(Recibido el 23-07-07, aceptado el 3-04-08) 